\title{
Unravelling adsorption and alignment of amyloid fibrils at interfaces by probe particle tracking $\dagger$
}

\author{
Lucio Isa, ${ }^{a}{ }^{J i n}-M i$ Jung $^{b}$ and Raffaele Mezzenga ${ }^{* c}$
}

\begin{abstract}
We report the first direct, non-invasive experimental evidence of a $2 \mathrm{D}$ isotropic-nematic transition for highly anisotropic nanoparticles at liquid-liquid interfaces by using passive fluorescent particle tracking. In order to illustrate the potential of this approach on systems of high real practical and biological relevance, we select as a model anisotropic nanoparticles $\beta$-lactoglobulin amyloid fibrils of varying aspect ratios. Upon nanoparticle adsorption at the interface, we follow, in real time and as a function of fibril length and bulk concentration, the development of a $2 \mathrm{D}$ nematic phase by studying the anisotropy in probe particle traces. We furthermore demonstrate the long-range nature of the nematic phase by calculating order parameters for the traces as high as 0.8 over $10^{2}$ to $10^{3} \mu \mathrm{m}^{2}$ areas. The presented route is independent of the system investigated, and thus these findings open a new, general strategy for the experimental assessment of 2D structural changes at anisotropic fluid-fluid interfaces.
\end{abstract}

\section{Introduction}

The alignment of high aspect ratio molecules and particles confined in two-dimensions (2D) has direct relevance in everyday life phenomena and applications such as liquid crystal displays, paints, sensors or biomimetic functions. ${ }^{1-3}$ Despite the great advances in the theoretical understanding of this process over the last four decades, ${ }^{4-7}$ the corresponding experimental progress has been remarkably slow. ${ }^{8}$ As per today, the establishment of a robust experimental route to the detection of two-dimensional structural changes at fluid interfaces, such as the 2D isotropicnematic transition, which is at the same time generally applicable and non-invasive, has yet to be found. Current experimental techniques have relied most frequently on indirect observations of the variations in the global surface properties associated with the orientation of anisotropic molecules or nanoparticles - also referred to as mesogenic units-confined in 2D geometries. Examples include ellipsometry, ${ }^{9}$ optical second harmonic generation, ${ }^{10}$ Brewster angle microscopy, ${ }^{11,12}$ interfacial birefringence, ${ }^{3} \mathrm{UV}$ adsorption spectroscopy ${ }^{13}$ or interfacial

${ }^{a}$ ETH Zurich, Laboratory for Surface Science and Technology, Wolfgang-Pauli-Strasse 10,8093 Zürich, Switzerland.E-mail: lucio.isa@, mat.ethz.ch

${ }^{b}$ Department of Physics, University of Fribourg, Ch. Musée 3, CH-1700 Fribourg, Switzerland. E-mail: Jin-Mi.Jung@rdls.nestle.com

${ }^{c}$ ETH Zurich, Food and Soft Materials Science, Institute of Food, Nutrition \& Health, Schmelzbergstrasse 9, 8092 Zurich, Switzerland. E-mail: raffaele.mezzenga@agrl.ethz.ch

$\uparrow$ Electronic supplementary information (ESI) available: Procedure for the nematic order parameter calculations and additional images of traces; rheology. ${ }^{14}$ Being ensemble techniques, however, these methods are not able to resolve local phenomena such as the presence of structural heterogeneities on the nanometre and micron length scales. For example, they cannot distinguish a pure nematic phase from the coexistence of isotropic and nematic domains which is predicted to appear under specific conditions. ${ }^{7}$

Other methods, such as Langmuir-Blodgett film deposition, do allow real-space characterization, but are also highly invasive, since they require the transfer of a monolayer from a fluid-fluid interface to a solid substrate. ${ }^{11,12,15}$ Two-dimensional organization has also been observed for rod-like virus composite films obtained via layer-by-layer deposition, although in this case the visualization remains limited to AFM on solid substrates. ${ }^{16}$ Finally, for those rare examples in which a direct in situ visualization of the orientation of the particles at the interface has been possible, either macroscopic objects in quasi-two dimensional geometries $^{17,18}$ or highly specific systems ${ }^{19,20}$ were used, so that a generalization of the corresponding experimental findings remains very limited.

\section{Results and discussion}

Particle tracking offers a unique combination of time-resolved, system-independent and non-invasive features, making it an unchallenged candidate to quantify, in situ and at small length scales, structural, dynamical and viscoelastic properties. ${ }^{21}$ The method is well established to study quiescent ${ }^{22}$ and driven ${ }^{23}$ bulk systems but it is still in its infancy to probe interfacial phenomena. ${ }^{24}$ In what follows we monitor the motion of fluorescent tracers (silica $r \approx 400 \mathrm{~nm}$ ) at a water/oil interface to investigate the irreversible adsorption ${ }^{25,26}$ of anisotropic, 
unlabelled $\beta$-lactoglobulin amyloid fibrils. Structural changes in the interfacial, self-assembled nanoparticles monolayer are revealed by detecting topological variations in the probe particle traces. The choice of amyloid fibrils as model anisotropic nanoparticles is dictated by their paramount importance in biological, medical and food science applications. Amyloid fibrils are protein aggregates, which can occur in vivo and are implicated in protein misfolding disorders such as Alzheimer's, CreutzfeldtJakob, Parkinson's or Huntington's disease ${ }^{27-30}$ and therefore their migration/adsorption at biological interfaces is a topic itself of crucial importance. Moreover, amyloid fibrils can also be prepared in vitro from harmless or food-grade proteins ${ }^{31}$ and thus they also bear great relevance in edible formulations or as food stabilizers due to their remarkable surface activity. ${ }^{14}$ Among the many fibril-generating food-grade proteins, $\beta$-lactoglobulin is particularly important. The adsorption of native $\beta$-lactoglobulin at oil/water and water/air interfaces has been assessed in numerous studies using different techniques and under different $\mathrm{pH}$, ionic strength and competitive adsorption conditions. The literature on the structural and rheological properties of native $\beta$-lactoglobulin interfacial layers is extremely vast (several hundred papers) and has been reviewed in detail by Dickinson ${ }^{32}$ and more recently by Murray. ${ }^{33}$ Despite the massive work on the adsorption of native $\beta$-lactoglobulin at liquid interfaces, the study of the behavior of $\beta$-lactoglobulin fibrils at interfaces is however in its infancy. In particular, in a previous work we have tackled the macroscopic interfacial rheology of $\beta$-lactoglobulin fibrils at interfaces, ${ }^{14}$ but detailed information on the microscopic behavior is currently missing. In this work we apply for the first time probe particle tracking to the case of $\beta$-lactoglobulin amyloid fibrils adsorbing at oil-water interfaces with the aim of elucidating the occurrence of a two-dimensional isotropicnematic transition and hint towards the generality of this method as a precious tool to test in situ and in a non-invasive manner the structural properties of interfacial assemblies of anisotropic nano- and microscale objects. To this end, we use here $\beta$-lactoglobulin amyloid fibrils of less than $10 \mathrm{~nm}$ in diameter and contour lengths varying from $10^{2} \mathrm{~nm}$ to $10^{4} \mathrm{~nm}$, so to give access to a broad range of nanoparticle aspect ratios. ${ }^{31,34,35} \mathrm{We}$ focus on two kinds of fibrils hereby referred to as long (Fig. 1a, 1-10 $\mu \mathrm{m}$ ) and short (Fig. 1b, 50-300 nm). Both fibril types are suspended in a pH 2 water solution in which they acquire a high positive linear charge density (monomer charge is $+20 \mathrm{e}^{-}$at $\mathrm{pH} 2$ ). ${ }^{34} \mathrm{We}$ point out that due to the fact that both the tracers and the proteins exhibit a strong positive charge at $\mathrm{pH} 2$ (see Methods section), adsorption and anchoring of proteins onto the probe particles are to be excluded due to electrostatic repulsion. In support of this expectation, particle-protein flocculation and precipitation were never observed at $\mathrm{pH} 2$ over several weeks. Moreover, even in the absence of the electrostatic repulsion dominating the present fibril/tracer system, high surface coverage of the colloidal tracers by the long fibrils is highly unlikely due to the mismatch between the particle size and the high rigidity and length of the fibrils (which have a persistence length $\geq 1 \mu \mathrm{m}$ and contour lengths $\left.\gg 1 \mu \mathrm{m}^{31}\right)$. A semi-flexible object with a persistence length larger than $1 \mu \mathrm{m}$ and $5-10 \mu \mathrm{m}$ contour length cannot easily adsorb onto nanoparticles with a curvature radius of $\sim 400 \mathrm{~nm}$, e.g. a third of the persistence length of the rod. ${ }^{36} \mathrm{In}$ agreement with these expectations, neither large-scale interfacial
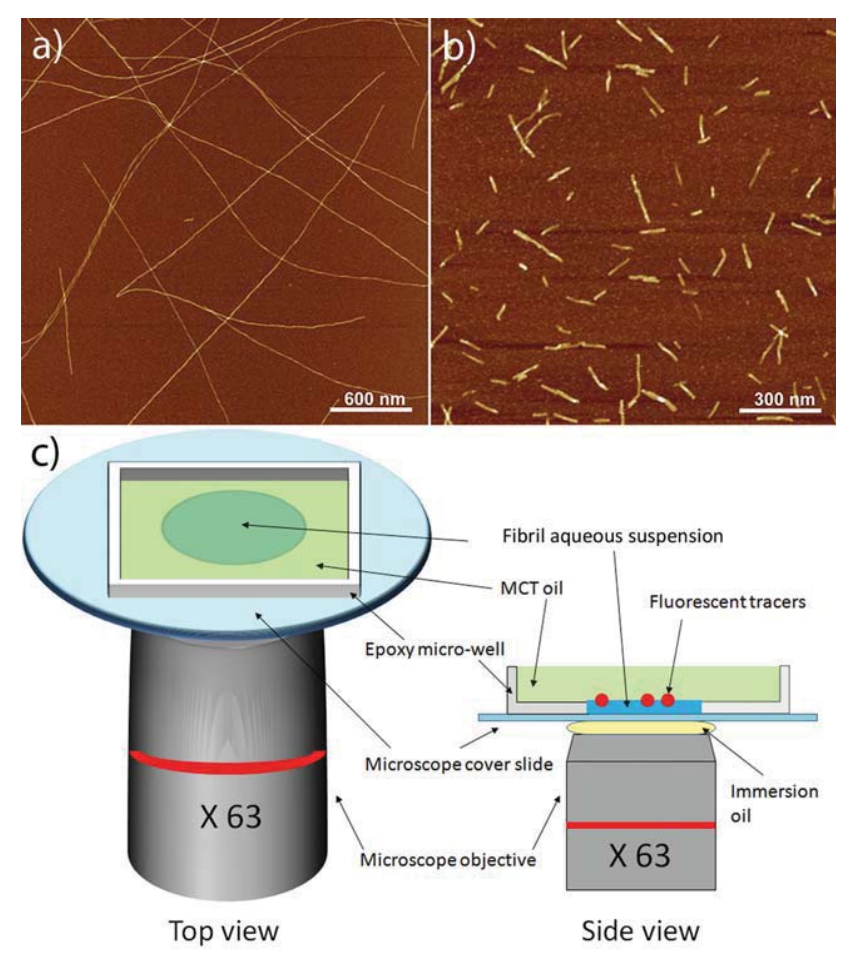

Fig. 1 AFM images of long (a) and short (b) $\beta$-lactoglobulin fibrils. (c) Schematics of the microscopy cell set-up used for interfacial particle tracking.

protein-particle aggregates nor correlated rigid-like motion of particle clusters (as it would be expected for fibrils bridging between particles) have ever been observed under the microscope, confirming the absence of fibril-particle coupling. Experiments at various bulk concentrations of fibrils $(c \leq 0.1 \%$ w/v) were carried out in a home-designed microscopy cell consisting of an epoxy resin micro-well of $1.5 \mathrm{~cm}$ diameter and $150 \mu \mathrm{m}$ thickness (Fig. 1c). By filling the micro-well up to the rim with the aqueous protein solution seeded with fluorescent tracers and subsequently carefully pouring medium chain triglycerides (MCTs) oil on top, a flat liquid-liquid interface was produced at a depth that could be imaged in an inverted microscope. Images were grabbed as a function of waiting time $t_{\mathrm{w}}$ from interface creation (minimum $t_{\mathrm{w}}=2-3$ minutes). A small percentage of the silica tracers got trapped at the interface (area coverage approx. $0.1-0.3 \%$ ) while the rest sedimented towards the bottom cover slide. This allowed us to locate the interface easily and to distinguish the tracers that are moving within the interface from the ones in the bulk of the suspension. In the absence of any protein adsorbed at the interface, the tracers undergo twodimensional Brownian motion with a diffusion coefficient of $2.25 \pm 0.53 \times 10^{-10} \mathrm{~cm}^{2} \mathrm{~s}^{-1}$. In the inset of Fig. 2a, a typical fluorescence micrograph of the system is shown; the brighter particles are trapped at the interface and only move in two dimensions, while the dimmer ones are dispersed below the interface and undergo three-dimensional thermal diffusion. Both species of particles can be tracked and a representative outcome of the tracking procedure is shown in Fig. 2a for a suspension of long fibrils with a bulk concentration of $0.1 \%$ and for $t_{\mathrm{w}}=95$ minutes. The first striking evidence is that the motion between 


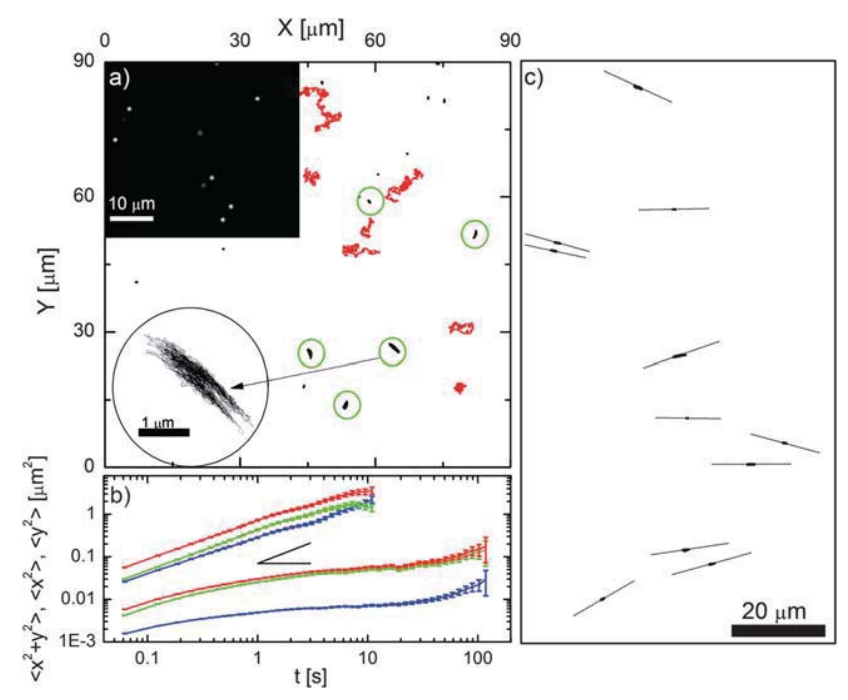

Fig. 2 (a) Example of particle traces at the interface (black) and below the interface (red) for a $0.1 \%$ long fibrils suspension at $\mathrm{pH} 2$ for $t_{\mathrm{w}}=$ 95 minutes. In the green circles the traces with strong 1D motion are highlighted and one of them is enlarged. Traces at the interface have been collected over 2000 frames while the ones in the bulk for a maximum of 400 frames due to loss of focus at longer times. Inset to (a): typical fluorescence micrograph of the system. The brighter tracers are at the interface, the dimmer ones are those moving in bulk. (b) Mean square displacements (msds) versus time of the same tracers below the interface (top three curves) and at the interface (bottom three curves). The total msds (red) have been decomposed along the main direction of motion $(x$, green) and the transverse direction ( $y$, blue). The solid lines highlight slope 1 (diffusive behaviour). (c) Example of high order 2D nematic phase, as revealed by the particle traces. The solid lines depict motion directions.

particles in the bulk (red) and at the interface (black) is very different; the two-dimensional projection of the 3D Brownian motion of the particles in the bulk shows much higher mobility with respect to the $2 \mathrm{D}$ motion at the interface. The difference in the mean square displacements (msds) is way too large to be solely explained by dimensionality-msds in Brownian-diffusion grow proportionally with time $t$ as $6 D t$ and $4 D t$, in $3 \mathrm{D}$ and $2 \mathrm{D}$ respectively, $D$ being the diffusion coefficient-but is to be ascribed to the different viscoelastic properties of the bulk and the interface. Secondly, it can be observed that while the motion in the bulk is isotropic and homogeneous, the situation is completely reversed at the interface. Here a high degree of heterogeneity in the particle mobility can be observed, and more importantly, strong anisotropy appears for some traces (Fig. 2a). This anisotropy can be further quantified by expressing the tracer msds as a function of time. Fig. $2 b$ compares the msds for the tracers in the bulk (top curves) to the ones at the interface (bottom curves). The total msds (red) have been decomposed along the main direction of motion ( $x$, green) and the transverse one ( $y$, blue). In the case of the particles in the bulk, it can be observed that they exhibit diffusive behaviour $(\mathrm{msd} \approx t)$ and that $x$ and $y$ motion contribute equally to the total msd. For the particles at the interface, the tracers show strongly sub-diffusive motion and the total displacement is to be almost completely addressed to $x$-motion. We ascribe the occurrence of strong anisotropy in the traces to the formation of nematic domains of fibrils at the interface, which force the particles to move along the direction of fibril alignment. It should be pointed out that the heterogeneity and anisotropy of the tracer motion cannot be extracted solely by ensemble quantities such as the msds; conversely, the possibility to resolve single particle traces offers the unique opportunity to probe structural details of the interface at length scales comparable with the probing particle size. As a further demonstration of the information that can be extracted from single particle traces, Fig. 2c shows an overlay of the traces with a line indicating the respective principal direction of motion. A high collective orientation of the one-dimensional diffusion traces can be preserved for distances as large as $100 \mu \mathrm{m}$ or more. From all the observedorientations of the traces one can extract a two-dimensional nematic order parameter of the particle traces, defined as $S_{2 \mathrm{D}}=\left\langle 2 \cos ^{2} \Theta-1\right\rangle$, where $\Theta$ is the angle between any individual trace direction and the mean orientation director $\eta$, and the brackets denote an average over all traces (more details on the procedure and more examples are given in the ESI $\dagger$ ). For the traces shown in Fig. 2c, a nematic order parameter as high as 0.78 has been calculated; by averaging over several locations in the same interface $(c=0.1 \%)$ a $S_{2 \mathrm{D}}$ of $0.59 \pm$ 0.06 is obtained. This trend, together with the occurrence of isotropic traces co-existing with one-directional ones (Fig. 2a), can be related to the presence of local isotropic domains in co-existence with nematic domains, which is expected from theoretical predictions ${ }^{7}$ but was not observed experimentally yet in situ. It should be stressed out that the definition of a twodimensional order parameter used here refers to static conditions, i.e. for short time scales relative to the dynamics of the system. Specifically, the time scale for the $2 \mathrm{D}$ order parameter calculation corresponds to trace acquisition times of 10-100 s (see Fig. 2b) in mature interfaces after much longer adsorption times (e.g. $t_{\mathrm{w}}=95$ minutes for Fig. $2 \mathrm{c}$ ). At these same conditions, our previous work ${ }^{14}$ showed that the interface is highly elastic (interfacial shear modulus dominating the interfacial loss modulus) with a complex shear modulus practically independent of the shear frequency at the time scales considered (10-100 s). This demonstrates unambiguously that at the experimental time scales of interest, the dynamics of the interface is essentially arrested by the elasticity of the protein film; therefore the time window used to acquire the probe particle traces is suitable to calculate a time-averaged 2D order parameter for the probe trajectories.

The effects of the bulk concentration, the aspect ratio of the fibrils and the adsorption kinetics can also be conveniently studied and disentangled. Fig. 3 reports the msds versus time for three different concentrations of long fibrils (top row) and three different concentrations of short fibrils (bottom row) at different adsorption lag times. For each case a representative trace is presented and related to the structure of the interface. Starting from the long fibrils, at a bulk concentration of $0.001 \%$ (Fig. 3a) it can be observed that, within the experimental time window considered, the tracer particles always undergo isotropic motion. At short adsorption times, diffusive motion with a diffusion coefficient of $2.75 \pm 0.50 \times 10^{-10} \mathrm{~cm}^{2} \mathrm{~s}^{-1}$ is observed. Upon raising the bulk concentration to $0.0033 \%$, the situation changes: at short times tracer motion is isotropic and diffusive, but after approximately 25 minutes, as the interfacial fibril concentration increases, a small number of traces develop anisotropy 

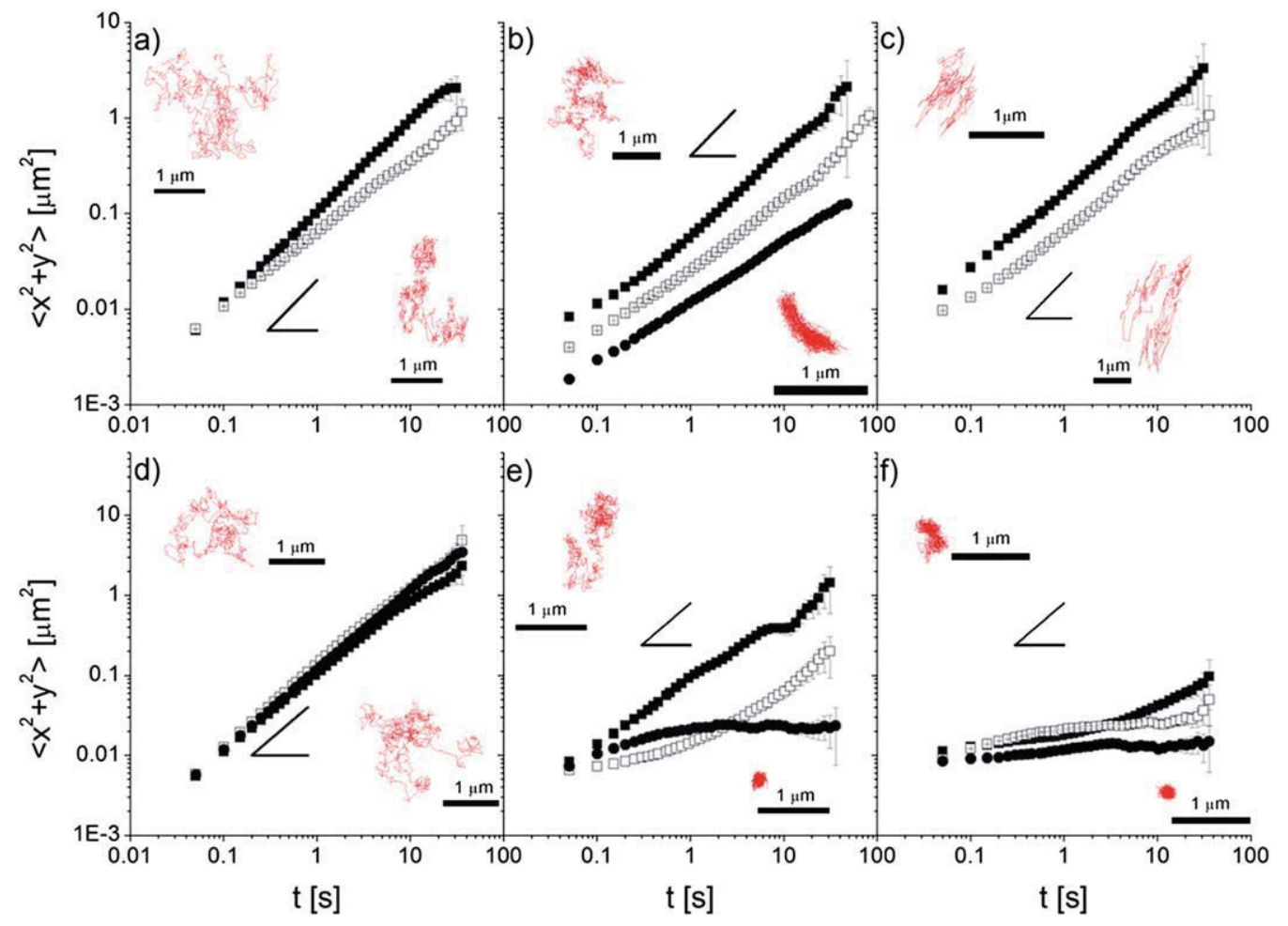

Fig. 3 Tracer msds versus time for different bulk concentrations of fibrils (top row: long fibrils; bottom row: short fibrils). A representative track is highlighted as inset at short (top left) and long times (bottom right) for each concentration and fibril type. (a) Long fibrils at a bulk concentration of $0.001 \%$ for: $\square t_{\mathrm{w}}=6$ minutes; $\square t_{\mathrm{w}}=28$ minutes. (b) Long fibrils at a bulk concentration of $0.0033 \%$ for: $\square t_{\mathrm{w}}=4$ minutes; $\square t_{\mathrm{w}}=13$ minutes; $t_{\mathrm{w}}=$ 25 minutes (concentration for which the IN transition is observed); (c) long fibrils at a bulk concentration of $0.01 \%$ for: $\mathbf{\square} t_{\mathrm{w}}=5$ minutes; $\square t_{\mathrm{w}}=$ 13 minutes. (d) Short fibrils at a bulk concentration of $0.001 \%$ for: $\quad t_{\mathrm{w}}=10$ minutes; $\square t_{\mathrm{w}}=16$ minutes; $t_{\mathrm{w}}=26$ minutes. (e) Short fibrils at a bulk concentration of $0.01 \%$ for: $\square t_{\mathrm{w}}=2$ minutes; $\square t_{\mathrm{w}}=14$ minutes; $t_{\mathrm{w}}=35$ minutes. (f) Short fibrils at a bulk concentration of $0.1 \%$ for: $\boldsymbol{\square} t_{\mathrm{w}}=$ 4 minutes; $\square t_{\mathrm{w}}=20$ minutes; $-t_{\mathrm{w}}=42$ minutes. In each graph the solid line slope represents diffusive behaviour.

(see bottom trace in Fig. 3b). Overall the tracer dynamics remains nearly purely diffusive, but heterogeneities do appear, indicating the formation of local islands of nematic order ( size $\approx 10^{2} \mu \mathrm{m}^{2}$ ). At even higher bulk concentrations $(0.01 \%$, Fig. 3c) nematic islands are formed immediately upon interface creation and anisotropy grows with time. At the highest bulk concentration investigated for the long fibrils, $0.1 \%$ shown in Fig. 2, interfacial diffusion becomes strongly sub-diffusive and it is accompanied by long-range nematic order. The situation is different for the short fibrils (Fig. 3d-f): all the tracers always show isotropic motion for all bulk concentrations. As an effect of increasing bulk concentration and adsorption times, the main change observed is a transition from a dilute isotropic interfacial assembly in which tracers freely diffuse (with a diffusion coefficient of $2.93 \pm 0.50 \times 10^{-10} \mathrm{~cm}^{2} \mathrm{~s}^{-1}$ ) to a strongly viscoelastic layer in which tracers are strongly caged, yet moving locally in a isotropic fashion. The fact that the diffusion coefficients for long and short fibrils at $c=0.001 \%$ and short waiting times coincide within the errors, and that they are very close to the value for the bare interface, is yet additional proof that anchoring of proteins on the tracers' surface does not play an important role in our case and that protein-particle interactions do not affect strongly tracer motion. The slightly larger values in the presence of proteins may be ascribed to a speeding up of diffusion due to the repulsive interactions among tracer particles and fibrils. At higher concentrations, when fibrils start to interact significantly, the structural and viscoelastic properties of the interfacial assemblies dominate the motion of the probe particles.

We point out that changing the bulk concentration has the primary effect of modifying the adsorption rate of the fibrils; the interplay between the interfacial protein concentration and the rate at which new fibrils adsorb and rearrange at the interface is responsible for the determination of the observed structure at any given time. For bulk concentrations of $0.001 \%$, the isotropic motion of the tracers highlights the fact that the interfacial concentration is too low to trigger fibril alignment within the experimental time window. Upon increasing the bulk concentration, the adsorption rate is sufficiently high to produce an interfacial concentration which allows for the observation of an alignment transition within our observation time.

The absence of a 2D IN transition for the short fibrils is consistent with considerations based on their reduced aspect ratio compared to longer fibrils and with corresponding results observed in the homologue three-dimensional case. ${ }^{35}$ The bulk IN transition for short $\beta$-lactoglobulin fibrils occurs at concentrations one order of magnitude larger (3-4\%) than for the long fibrils $(0.3-0.4 \%)$. This also explains why all the particle traces observed in bulk at the concentrations explored here always have an isotropic path. In the case of a system confined in two dimensions the additional geometrical constraints in the 
excluded volume imply that at the interfacial concentration necessary for the 2D IN transition to take place, the system may already be frozen in a disordered, isotropic network of fibrils. The scenario changes for the long fibrils; the 2D IN transition happens at much lower concentrations due to their larger aspect ratio, so it is possible for the fibrils to rearrange into nematic domains at the interface upon adsorption, before a viscoelastic interface builds up. Clearly, heterogeneities in the fibril arrangement translate into heterogeneity in the tracer motion, with some tracers locally trapped in small cages, others sampling larger areas and others forced to follow a one-dimensional motion within nematic domains. In order to explore these aspects further we have built spatial maps of the anisotropy of particle motion for the long fibrils at a bulk concentration of $0.1 \%$ and two examples are shown in Fig. 4, where the mean ratio of the main and transverse msd over 200 frames is plotted for each tracer at the interface. We notice immediately that the tracer behavior is highly heterogeneous with a certain number of particles moving isotropically (triangles) and others showing strongly anisotropic motion (squares). The values between the tracers have been interpolated to give a visual representation of domains in which the motion of the probe particles is strongly influenced by the local fibril alignment. By comparing Fig. 4a and $b$, we also see that in different locations on the same interface the size of these anisotropic domains is also heterogeneous. Heterogeneities for interfacial layers of native proteins at interfaces have already been resolved by particle tracking ${ }^{37}$ and confocal microscopy. ${ }^{38}$ In the case of native proteins, these heterogeneities have been interpreted in terms of intermolecular cross-linking of proteins and predicted by numerical simulations. ${ }^{39}$ In the present case, however, the physical origin of these heterogeneities is very different since they result from the local excluded volume associated with the fibrils. The present work thus provides the first non-invasive experimental mapping of the coexisting 2D isotropic and nematic domains generated by the adsorption of stiff, long protein fibrils at liquid-liquid interfaces. Since fibril dimensions set a limit to the maximum observable mesh size of the interfacial protein assembly, for the short fibrils the size of the structural heterogeneities is too small compared to the tracer size to be resolved and therefore, at high enough interfacial concentrations, the probe particles are effectively caged in a strong, uniform viscoelastic matrix. Such conclusion is further supported by Fig. 5 which compares the tracer msds for $c=0.1 \%$ of long fibrils, short fibrils and $\beta$-lactoglobulin monomers as a function of adsorption time $t_{\mathrm{w}}$. For all cases we observe a slowing down of the tracer dynamics upon protein adsorption with strong caging at long times for the monomers and the short fibrils, while the long fibrils still allow for sub-diffusive tracer dynamics, given the larger mesh size in the interfacial assembly and the presence of anisotropic motion within nematic domains. In other words, the size of the tracers determines the length scale of the structural features that can be probed; tracers of a size intermediate between the length of the long and short fibrils are sensitive to features in the micron range but are blind to structural details on the sub-500 nm length scale. Therefore, in the case of the short fibrils, probe particles only yield a mean effective response, averaged over many structural units. In order to elucidate this point further, we have analysed our data by decomposing tracer motion along the principal $(x)$ and transverse $(y)$ direction of motion in a local, particle-dependent, co-moving frame of reference, as already described in Fig. 2c, as a function of fibril length and bulk concentration. To clarify the role played by tracer size we have also performed experiments with larger tracers $(5.4 \mu \mathrm{m}$ diameter melamine-formaldehyde (MF) resin particles). The results are summarized in Table 1, which reports the ratio of the msds $\left\langle\Delta x^{2}\right\rangle /\left\langle\Delta y^{2}\right\rangle$ at a time $t=10 \mathrm{~s}$ (corresponding to 200 frames, for which very good statistics is obtained). As anticipated, for the long fibrils at low bulk concentrations $(c=0.001 \%)$ the motion of the silica tracers is isotropic within the error, while it develops strong anisotropy at high bulk concentrations $(c=0.1 \%)$. The larger spread in the values for $c=0.1 \%$ is to be ascribed to the heterogeneous behaviour at the interface as previously discussed. When looking at the short fibrils, the motion of the silica tracers remains isotropic at all concentrations underlining the size-dependence of the probe particle relative to the length scale of the structure.
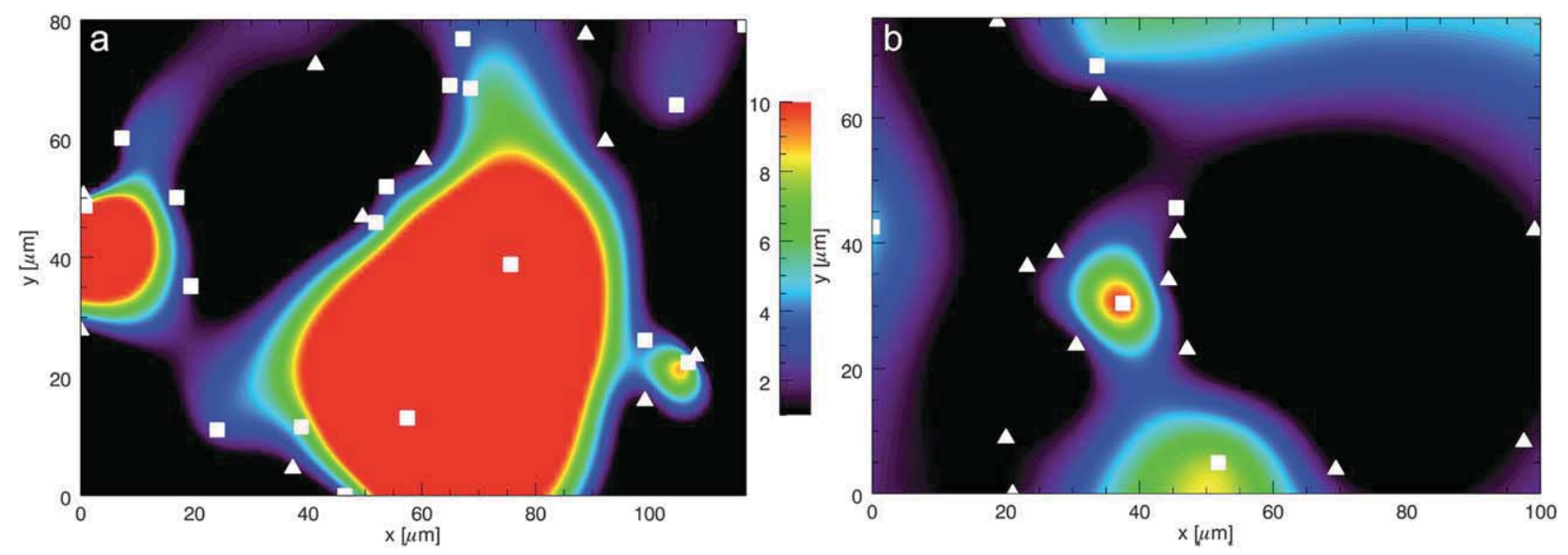

Fig. 4 Spatial maps of tracer anisotropy $\left\langle\Delta x^{2}\right\rangle /\left\langle\Delta y^{2}\right\rangle$ at the two different positions at the same interface for long fibrils with a bulk concentration $c=$ $0.1 \%$ and $t_{\mathrm{w}}>90$ minutes. The symbols represent the average position of the tracers, whose motion is distinguished between strongly anisotropic $\left(\left\langle\Delta x^{2}\right\rangle /\right.$ $\left\langle\Delta y^{2}\right\rangle$ greater than $\left.1.5, \mathbf{\square}\right)$ and weakly anisotropic or isotropic $\left(\left\langle\Delta x^{2}\right\rangle /\left\langle\Delta y^{2}\right\rangle\right.$ less than $\left.1.5, \boldsymbol{\Delta}\right)$. The values between the tracers have been interpolated to visualize regions of similar tracer motion. 


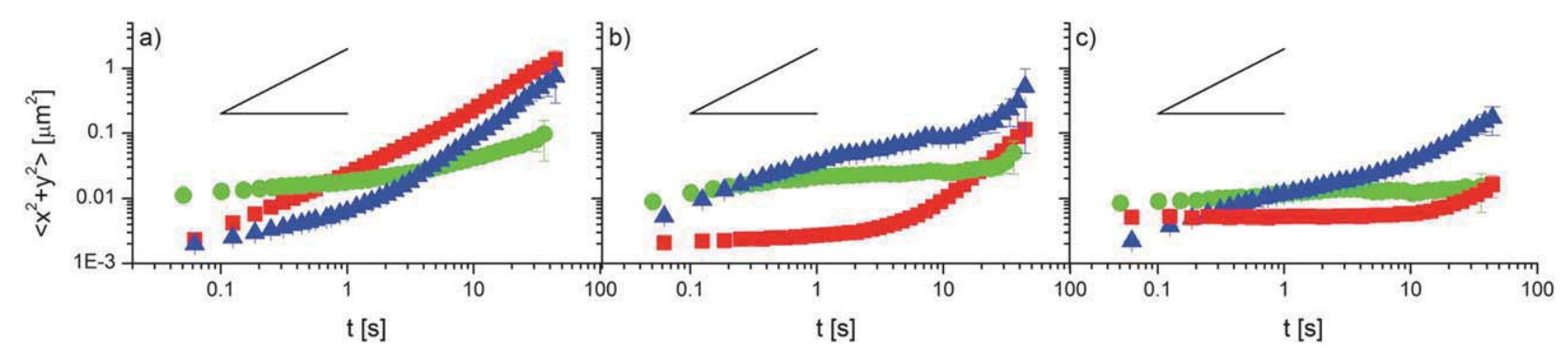

Fig. 5 Mean square displacements $v s$ time for tracer particles at the interface with a bulk concentration $c=0.1 \%$ for monomers $(\boldsymbol{\square})$, short fibrils $(\boldsymbol{O})$ and long fibrils ( $\boldsymbol{\Delta}$ ). (a) Short times: monomers $t_{\mathrm{w}}=3$ minutes; short fibrils $t_{\mathrm{w}}=4$ minutes; long fibrils $t_{\mathrm{w}}=4$ minutes. (b) Intermediate times: monomers $t_{\mathrm{w}}=16$ minutes; short fibrils $t_{\mathrm{w}}=19$ minutes; long fibrils $t_{\mathrm{w}}=14$ minutes. (c) Long times: monomers $t_{\mathrm{w}}=30$ minutes; short fibrils $t_{\mathrm{w}}=40$ minutes; long fibrils $t_{\mathrm{w}}=32$ minutes.

Table 1 Summary of tracer anisotropy for different tracers, fibril types and bulk concentrations. The ratio of msds in the principal and transverse direction of motion has been calculated by decomposing particle motion in a local, particle-dependent co-moving reference frame and it has been averaged over 200 frames corresponding to $10 \mathrm{~s}$ of live imaging.

\begin{tabular}{|c|c|c|c|}
\hline Fibril/tracer type & Bulk concentration & $\frac{\left\langle\Delta x^{2}\right\rangle}{\left\langle\Delta y^{2}\right\rangle_{t=10 s}}$ & $\begin{array}{l}\text { Error } \\
\text { (standard deviation) }\end{array}$ \\
\hline Long fibrils/silica tracers & $0.001 \%$ & 1.17 & \pm 0.17 \\
\hline Long fibrils/silica tracers & $0.1 \%$ & 5.49 & \pm 1.08 \\
\hline Long fibrils/MF tracers & $0.1 \%$ & 1.12 & \pm 0.03 \\
\hline Short fibrils/silica tracers & $0.001 \%$ & 1.19 & \pm 0.18 \\
\hline Short fibrils/silica tracers & $0.1 \%$ & 1.07 & \pm 0.07 \\
\hline
\end{tabular}

Such limitation in the probe spatial resolution is even more convincingly highlighted by noting that the motion of the larger MF tracers becomes practically isotropic even when the smaller silica tracers show strong anisotropy, underlining how the tracer size can mask the structural features of the interface. The choice of different probe types can thus be exploited as a way to characterize the interfacial properties over several length scales and dynamical regimes. This fact also becomes apparent when comparing our results to the anisotropy in tracer motion as observed in bulk nematics. ${ }^{40,41}$ In the case of the long fibrils, the aspect ratio between the probe particles and the anisotropic objects is reversed compared to bulk nematics. As a consequence of this, the observed anisotropy in tracer motion is much higher than that observed for small molecules or short rods in bulk. By increasing the probe size or using short fibrils, the aspect ratios approach the ones found in the bulk cases, but, as described above, no marked anisotropy is observed. The difference may stem from the fact that in bulk the tracers are fully immersed in a nematic environment, while at the interface they only see a $\sim 10 \mathrm{~nm}$ thick anisotropic sheet. Therefore, unless an extremely strong 2D anisotropic environment is present, as in the case of the long, stiff fibrils, anisotropy in the 2D particle motion appears to be weaker than in the $3 \mathrm{D}$ counterpart. Once more, the technique's sensitivity can be improved by reducing the tracer size and thus detect anisotropy on smaller length scales.

\section{Conclusions}

In summary, we have presented a new strategy to follow structural changes at fluid-fluid interfaces, in a non-invasive way, in situ, and at length scales comparable to the probe particle size. In particular the non-invasive character of our method is confirmed by the fact that the typical size of the structural domains found in the system greatly exceeds $\left(\sim 10^{2} \mu \mathrm{m}\right)$ the probe particle size $(\sim 750 \mathrm{~nm})$. Moreover the average distance between tracer particles at the interface is also much larger than the length (and thickness) of the fibrils. Therefore, even if the adsorption and fibril orientation in the immediate vicinity of a tracer were affected by the presence of the latter, the overall structure of the interface would not be disturbed by the probes. Moreover, the surface properties of the probes were chosen to avoid anchoring and minimize interactions with the proteins. Although the potential of this approach has been illustrated for the case of IN transitions of amyloid fibrils at fluid-fluid interfaces, the outcome remains general in terms of investigated systems and structural changes. The emergence of anisotropy in the probe traces, showing correlations over very large distances, provides convincing evidence of long-range nematic order for the long fibrils. Similarly, the reduction of particle mobility versus time for the monomers and short fibrils also demonstrates the capability of the method to observe the microscopic build-up of viscoelastic interfaces. ${ }^{37}$ By performing additional characterization, including measuring the contact angles of the tracers in the experimental conditions, one can further quantitatively extract local viscoelastic properties via a full microrheological treatment of the observable quantities. ${ }^{21,34,37,42}$ It can be anticipated that, if different tracer sizes are systematically exploited, various dynamical regimes and distinct length scales can be accessed, opening up the use of this technique to the study of the many diverse systems in nano- and biotechnology involving liquid-liquid and liquid-air interfaces. 


\section{Materials and methods}

\section{Fibrils preparation}

The long $\beta$-lactoglobulin fibrils were produced by heat denaturation of monomers at $\mathrm{pH} 2$ and $90{ }^{\circ} \mathrm{C}$ for 300 min following available protocols and previous literature reports. ${ }^{29-31}$ To obtain the short fibrils, a solution of long $\beta$-lactoglobulin fibrils was diluted to $1 \mathrm{wt} \%$ with $\mathrm{pH} 2$ Milli-Q water and homogenized at 700 bar through five iterative cycles using a two-stage highpressure homogenizer (Panda 2K, Niro Soavi, Italy). In order to remove unreacted monomers and small molecular weight surface active components coexisting with fibrils after their formation, both long and short fibrils suspensions were further dialyzed against pH 2 Milli-Q water, using a Cellulose Ester Spectra-Por dialysis membrane having a MWCO of 100000 Da (Spectrum Laboratories, Inc., CA, USA). The efficiency of the dialysis was estimated by measuring the concentration in the dialysis bath via absorbance measurements at $278 \mathrm{~nm}$.

\section{AFM imaging}

$10 \mu \mathrm{L}$ aliquots of $\beta$-lactoglobulin fibril solutions were diluted to $0.1 \mathrm{wt} \%$ at $\mathrm{pH} 2$ and deposited onto freshly cleaved mica, incubated for $2 \mathrm{~min}$, rinsed with Milli-Q water, and dried under nitrogen. Images were collected using a MultiModeV (Veeco Inc., Woodbury, NY, USA) operated in tapping mode in air.

\section{Microscopy and particle tracking}

The microscopy cells were prepared by stamping a PDMS replica into UV-curable epoxy resin (Norland Optical Adhesive 81, NJ, USA) on a $4 \mathrm{~cm}$ diameter \#1 round borosilicate glass cover slide (Thermo Scientific, Menzel-Gläser, Germany). Before fabrication, the cover slides were ultrasonicated in isopropanol and Milli-Q water (30 minutes each) and immediately before imaging they were exposed to 60 minutes of UV-ozone treatment (Boekel UV Cleaner 135500, PA, USA) in order to make the bottom of the micro-well highly hydrophilic. Fluorescent silica tracers (diameter $=774 \mathrm{~nm} \pm 90 \mathrm{~nm}$ from SEM, from Dr A. Schofield, The University of Edinburgh, UK) were added at $0.3 \% \mathrm{v} / \mathrm{w}$ in the water protein fibril suspensions. The surface modification of the silica tracers used to incorporate the fluorescent dye (FITC) renders the particle surface positively charged $(\zeta$ potential at $\mathrm{pH}$ $2=+80 \pm 2.5 \mathrm{mV}) .{ }^{43,44}$ Melamine-formaldehyde $(\mathrm{MF})$ resin particles (diameter $=5.41 \pm 0.12 \mu \mathrm{m}$, microparticles $\mathrm{GmBH}$, Germany) also have a positive charge at $\mathrm{pH} 2(+66.0 \pm 5.2 \mathrm{mV})$ and were added at $0.4 \% \mathrm{v} / \mathrm{w}$ in the water protein fibril suspensions. The particle $\zeta$ potentials were measured using a ZetaSizer instrument (Malvern, UK). Imaging was performed on a Zeiss 200M (Germany) inverted microscope using a $63 \times 1.4$ NA Oil DICIII Plan Apochromat lens. The collected images were analyzed using standard particle tracking techniques as well as custom-written software in IDL (ITT Visual Information Solutions, CO, USA). Prior to the msd calculations and trace representation, any drift present in the images was removed by a "bootstrap approach". ${ }^{23}$ This consists of a first PIV-like step to remove large advected motion and a second fine step where any residual sub-pixel drift is calculated from the total particle displacements and subsequently subtracted to the particle motion to yield a true co-moving reference frame for each individual particle.

\section{Acknowledgements}

The authors acknowledge A. B. Schofield for providing the silica tracers. E. Reimhult, M. Textor, N. D. Spencer, G. Csucs, O. Biehlmaier, J. Adamcik, C. Lara and the Light Microscopy Centre (LMC) at ETHZ are acknowledged for support and discussions. LI acknowledges financial support from MC-IEF2009-252926.

\section{References}

1 J. H. Kim, M. Yoneya and H. Yokoyama, Nature, 2002, 420, 159162.

2 M. Vilfan, A. Potocnik, B. Kavcic, N. Osterman, I. Poberaj, A. Vilfan and D. Babic, Proc. Natl. Acad. Sci. U. S. A., 2010, 107, 1844-1847.

3 M. I. Boamfa, M. W. Kim, J. C. Maan and T. Rasing, Nature, 2003, 421, 149-152.

4 P. G. De Gennes, The Physics of Liquid Crystals, Oxford University Press, 1974.

5 D. W. Berreman, Phys. Rev. Lett., 1972, 28, 1683-1686.

6 M. A. Bates and D. Frenkel, J. Chem. Phys., 2000, 112, 10034-10041.

7 R. L. C. Vink, Phys. Rev. Lett., 2007, 98, 217801.

8 B. Jerome, Rep. Prog. Phys., 1991, 54, 391-451.

9 S. Immerschitt, T. Koch, W. Stille and G. Strobl, J. Chem. Phys., 1992, 96, 6249-6256.

10 P. Guyotsionnest, H. Hsiung and Y. R. Shen, Phys. Rev. Lett., 1986, 57, 2963-2966.

11 M. P. Shortell, H. W. Liu, H. Y. Zhu, E. A. Jaatinen and E. R. Waclawik, Langmuir, 2010, 26, 14472-14478.

12 J. L. Hernandez-Lopez, E. R. Alvizo-Paez, S. E. Moya and J. RuizGarcia, J. Phys. Chem. B, 2006, 110, 23179-23191.

13 K. S. Yim, G. G. Fuller, A. Datko and C. D. Eisenbach, Macromolecules, 2001, 34, 6972-6977.

14 J. M. Jung, D. Z. Gunes and R. Mezzenga, Langmuir, 2010, 26, $15366-15375$.

15 F. Kim, S. Kwan, J. Akana and P. D. Yang, J. Am. Chem. Soc., 2001, 123, 4360-4361.

16 P. J. Yoo, K. T. Nam, J. F. Qi, S. K. Lee, J. Park, A. M. Belcher and P. T. Hammond, Nat. Mater., 2006, 5, 234-240.

17 J. Galanis, D. Harries, D. L. Sackett, W. Losert and R. Nossal, Phys. Rev. Lett., 2006, 96, 028002.

18 J. Galanis, R. Nossal, W. Losert and D. Harries, Phys. Rev. Lett., 2010, 105, 168001.

19 Z. Y. Zheng and Y. L. Han, J. Chem. Phys., 2010, 133, 124509.

20 Y. G. Li and Y. Y. Wu, J. Am. Chem. Soc., 2009, 131, 5851-5857.

21 T. G. Mason, K. Ganesan, J. H. vanZanten, D. Wirtz and S. C. Kuo, Phys. Rev. Lett., 1997, 79, 3282-3285.

22 V. Prasad, D. Semwogerere and E. R. Weeks, J. Phys.: Condens. Matter, 2007, 19, 113102.

23 R. Besseling, L. Isa, E. R. Weeks and W. C. K. Poon, Adv. Colloid Interface Sci., 2009, 146, 1-17.

24 F. Ortega, H. Ritacco and R. G. Rubio, Curr. Opin. Colloid Interface Sci. 2010, 15, 237-245.

25 C. J. Beverung, C. J. Radke and H. W. Blanch, Biophys. Chem., 1999, 81, 59-80.

26 E. M. Freer, K. S. Yim, G. G. Fuller and C. J. Radke, J. Phys. Chem. $B, 2004,108,3835-3844$.

27 R. J. Ellis and T. J. T. Pinheiro, Nature, 2002, 416, 483-484.

28 C. M. Dobson, Nature, 2003, 426, 884-890.

29 F. Chiti and C. M. Dobson, Annu. Rev. Biochem., 2006, 75, 333-366.

30 A. A. Cooper, A. D. Gitler, A. Cashikar, C. M. Haynes, K. J. Hill, B. Bhullar, K. N. Liu, K. X. Xu, K. E. Strathearn, F. Liu, S. S. Cao, K. A. Caldwell, G. A. Caldwell, G. Marsischky, R. D. Kolodner, J. LaBaer, J. C. Rochet, N. M. Bonini and S. Lindquist, Science, 2006, 313, 324-328.

31 J. Adamcik, J. M. Jung, J. Flakowski, P. De Los Rios, G. Dietler and R. Mezzenga, Nat. Nanotechnol., 2010, 5, 423-428.

32 E. Dickinson, Colloids Surf., B, 1999, 15, 161-176.

33 B. S. Murray, Curr. Opin. Colloid Interface Sci., 2011, 16, 27-35. 
34 J. M. Jung, G. Savin, M. Pouzot, C. Schmitt and R. Mezzenga, Biomacromolecules, 2008, 9, 2477-2486.

35 J. M. Jung and R. Mezzenga, Langmuir, 2010, 26, 504-514.

36 W. Z. Zhou, J. Cao, W. C. Liu and S. Stoyanov, Angew. Chem., Int Ed., 2009, 48, 378-381.

37 M. H. Lee, D. H. Reich, K. J. Stebe and R. L. Leheny, Langmuir, 2010, 26, 2650-2658.

38 R. Mezzenga, P. Schurtenberger, A. Burbidge and M. Michel, Nat. Mater., 2005, 4, 729-740.

39 C. M. Wijmans and E. Dickinson, Langmuir, 1998, 14, 7278-7286.
40 J. C. Loudet, P. Hanusse and P. Poulin, Science, 2004, 306, 1525.

41 A. A. Verhoeff, J. van Rijssel, V. W. A. de Villeneuve and H. N. W. Lekkerkerker, Soft Matter, 2008, 4, 1602-1604.

42 V. Prasad, S. A. Koehler and E. R. Weeks, Phys. Rev. Lett., 2006, 97, 176001.

43 K. A. White, A. B. Schofield, B. P. Binks and P. S. Clegg, J. Phys. Condens. Matter, 2008, 20, 494223.

44 K. A. White, A. B. Schofield, P. Wormald, J. W. Tavacoli, B. P. Binks and P. S. Clegg, J. Colloid Interface Sci., 2011, 359, 126-135. 